\section{Is sphenoid sinus opacity significant in patients with optic neuritis?}

CONSUELA M. MOORMAN, PHILIP ANSLOW, JOHN S. ELSTON

\section{C.M. Moorman \\ P. Anslow \\ J.S. Elston \\ Department of \\ Ophthalmology and \\ Neuroradiology \\ Radcliffe Infirmary \\ Oxford, UK}

Ms C.M. Moorman

1 Tackley Place

Oxford OX2 6RR, UK

Tel: $+44(0) 1865513851$

Fax: +44 (0)1865 224013

Received: 26 May 1998 Accepted in revised form: 16 July 1998

\begin{abstract}
Purpose Optic neuritis secondary to sinus disease is an infrequent but well-documented association. When a patient presents with signs of optic nerve dysfunction and orbital inflammation the significance of widespread sinus disease on radiology is clear and the management is straightforward. We present a group of patients with isolated optic neuritis and radiological evidence of sphenoethmoiditis and discuss the clinical relevance of this finding.

Methods We reviewed the notes of 11 patients with optic neuritis who, because of atypical headache, underwent neuroimaging revealing sphenoid sinus opacity. Six patients had endoscopic drainage of the sphenoid sinus; 4 were treated medically.

Results Sinus contents included fungal infection (2), mucopurulent material (5), polyps (1) and necrotic tumour (1). Narrowing of the optic canal due to chronic osteomyelitis was found in 1 patient with irreversible optic atrophy. Visual loss was reversible in 6 patients. Four patients had normal radiological findings after treatment. Two patients had recurrent optic neuritis with sphenoid sinusitis on MRI scan, resolving on treatment, during the 4 year follow-up period.

Conclusions Possible mechanisms of nerve damage in this situation include direct spread of infection, occlusive vasculitis and bony deficiency in the wall of the sinus. Patients presenting with isolated optic neuritis and atypical headache should be scanned; an opaque sphenoid sinus in the context of visual loss should not be dismissed as coincidental but assumed to be pathological and the patient referred for drainage. Sphenoid sinusitis is an uncommon but treatable cause of optic neuritis.
\end{abstract}

Key words Optic neuritis, Sinus disease, Sphenoid sinus opacity

Optic neuritis is defined as an inflammatory neuropathy of the optic nerve that presents with sudden unilateral visual loss. The affected eye characteristically has impaired colour vision, an afferent pupillary defect and visual field loss; the patient may also complain of painful eye movements and, if the inflammation is anterior, the optic disc is swollen. Patients typically recover vision in the affected eye over the subsequent 3-6 weeks. Review of the literature suggests that between $49 \%$ and $61 \%$ of patients with isolated optic neuritis have MRI

abnormalities consistent with multiple sclerosis at presentation. ${ }^{1,2}$ The precise aetiology in the remainder of patients is often undetermined. Optic neuropathy has been reported in association with various conditions including hypertension and hyperthyroidism, ${ }^{3}$ infections such as measles, rubella, mumps and infectious mononucleosis, ${ }^{4}$ systemic neoplasia, ${ }^{5}$ and poisoning from substances such as lead, arsenic and methanol. ${ }^{3,6}$

Optic neuritis secondary to paranasal sinus disease is an infrequent but well-documented association. ${ }^{3}$ It is evident, for example, in patients presenting with optic nerve dysfunction, orbital signs and spheno-ethmoid sinus infection on radiology ('orbital cellulitis'). The difficulty arises when a patient presents with isolated optic neuritis and is found to have radiological evidence of sphenoiditis. The close proximity of the optic nerve in the optic canal to the sphenoid sinus makes it vulnerable to direct spread of infection from the sphenoid sinus. There have been numerous case reports in the literature - both historical and contemporary which lend support to this view. ${ }^{7,8}$ To test the hypothesis that sphenoid sinusitis causes acute optic neuritis we analysed the clinical and radiological findings of 11 patients in whom this association had been demonstrated.

\section{Methods}

Using the diagnostic index at Oxford Eye Hospital we retrospectively reviewed the notes of all cases of optic neuritis presenting between September 1992 and December 1997. Diagnostic criteria included sudden unilateral visual loss with optic nerve dysfunction, visual field loss and improvement of vision within $4-6$ weeks. Most of the cases presented to the Eye Casualty 
Table 1. Clinical features of patients with optic neuritis and sphenoid sinus opacity

\begin{tabular}{lcccccl}
\hline $\begin{array}{l}\text { Patient } \\
\text { no. }\end{array}$ & $\begin{array}{c}\text { Age } \\
\text { (years) }\end{array}$ & $\begin{array}{c}\text { Initial } \\
\text { VA }\end{array}$ & $\begin{array}{c}\text { Final } \\
\text { VA }\end{array}$ & $\begin{array}{c}\text { Atypical features } \\
\text { of ON }\end{array}$ & Disc appearance & Pathology \\
\hline 1 & 10 & $6 / 12$ & $6 / 36$ & $\mathrm{H}+\mathrm{SD}$ & Pale & Pus. Positive culture \\
2 & 12 & $6 / 18$ & $6 / 5$ & $\mathrm{H}$ & Swollen & Medical treatment \\
3 & 12 & $\mathrm{CF}$ & $6 / 5$ & $\mathrm{H}$ & Swollen & Pus. Culture negative \\
4 & 28 & $6 / 9$ & $6 / 5$ & $\mathrm{H}+\mathrm{SD}$ & Normal & Medical treatment \\
5 & 37 & $6 / 12$ & $6 / 9$ & $\mathrm{H}$ & Normal & Pus. Culture negative \\
6 & 61 & $6 / 12$ & $6 / 9$ & $\mathrm{H}$ & Normal & Aspergillosis \\
7 & 63 & $\mathrm{CF}$ & $\mathrm{CF}$ & $\mathrm{H}+\mathrm{SD}$ & ?Swollen & Aspergillosis \\
8 & 66 & $6 / 18$ & $\mathrm{n} / \mathrm{a}$ & $\mathrm{H}$ & Normal & Sinus tumour \\
9 & 55 & $6 / 9$ & $6 / 6$ & $\mathrm{H}$ & Swollen & Medical treatment \\
10 & 58 & $6 / 18$ & $6 / 9$ & $\mathrm{H}$ & Swollen & Polyps \\
11 & 36 & $\mathrm{HM}$ & $6 / 18$ & $\mathrm{H}$ & Pale & No treatment \\
\hline
\end{tabular}

${ }^{a}$ Atypical features; $\mathrm{H}$, headache; $\mathrm{SD}$, systemic disturbance.

VA, visual acuity; $\mathrm{ON}$, optic neuritis; $\mathrm{CF}$, count fingers vision; $\mathrm{HM}$, hand movement vision.

and were referred to the out-patient clinic; $3 \%$ of cases were referred from other sources such as the Neurology Department. Patients with this diagnosis are not investigated routinely in our department unless there are atypical features such as age outside the normal for the condition or systemic symptoms. Of a total of 149 patients, 84 had CT or MRI scans, and of these $11(13 \%)$ were shown to have radiological evidence of sphenoid sinusitis (7 on MRI scan and 4 on CT scan). One of these patients subsequently developed clinical and radiological evidence of multiple sclerosis, leaving 10 with acute optic neuritis and isolated sphenoid sinus opacity.

All 11 patients in the study had been scanned because they had one or more of the following features: atypical headache (11 patients), systemic symptoms such as fever or nausea (3 patients), unusual age at presentation, i.e. under 18 years ( 3 patients) or over 50 years ( 5 patients). Patients described their headache, distinct from retrobulbar pain or pain associated with eye movements, as moderate to severe and located to the vertex or occipital region of the skull in 8 cases.

\section{Results (Table 1)}

Six females and five males ranging in age from 10 to 66 years were identified and their records examined. All patients presented with reduced visual acuity associated, in 5 cases, with some degree of retrobulbar discomfort

\section{Table 2. Summary of patient outcome after treatment}

\begin{aligned} & \hline $\begin{array}{l}\text { Patient } \\ \text { no. }\end{array}$ Outcome \\ & \hline 1 Vision stabilised. On partial sight register. Normal MRI \\ & 2 Normal vision. One recurrence. Resolved. Normal MRI \\ & 3 Normal vision. Normal MRI \\ & 4 Normal vision. Normal MRI \\ & 5 Vision stabilised. Persistent headaches \\ & 6 Recovery of vision \\ & 7 No recovery. Developed ophthalmoplegia. Died \\ & 8 Radiotherapy. Lost to follow-up \\ & 9 Normal vision \\ & 10 Normal vision \\ & 11 Partial recovery. MRI suggestive of multiple sclerosis \\ & \hline\end{aligned}

and, in 8 eyes, with an afferent pupillary defect and reduced colour vision on Ishihara testing. In addition to these typical features of optic neuritis all 11 patients complained of moderate to severe headache, localised to the vertex in 8 patients, and 3 of them experienced nausea, sweating attacks and general malaise.

All patients were referred to the ENT surgeons for an opinion. Endoscopic drainage of the posterior sphenoethmoid sinuses was advised in 7 whilst a further 3 patients were treated medically with intravenous antibiotics. At operation the sinus opacity was found to be caused by mucopurulent material in 5 cases; cultures were positive for Aspergillus fumigatus in 2 patients, Staphylococcus aureus in 1 patient and negative in the rest. One patient was found to have polyps; cultures were positive for Staphyloccus epidermidis and the final patient was found to have a necrotic tumour filling the sphenoid sinus cavity. The subsequent clinical course varied (Table 2).

Patient 1, a 10-year-old girl, initially presented in 1994 with unilateral optic neuropathy (decreased vision, RAPD and a pale atrophic disc). Neuro-imaging at that time revealed inflammation of the left optic nerve; an opaque sphenoid sinus was also noted but felt to be incidental (Fig. 1a). Two years later she developed recurrent attacks of optic neuritis in the fellow eye and described episodes of visual loss accompanied by nausea, fever and headache. Repeat imaging revealed a persistent sphenoid sinus opacity and in addition marked narrowing of the optic canals was noted due to extensive, chronic osteomyelitis of the bony wall of the sinus (Fig. 1b-d). After drainage and treatment with broad spectrum antibiotics, vision on the right eye stabilised but the compressive neuropathy of the left optic nerve resulted in permanent visual loss.

Visual loss was fully reversible in patients 2, 3, 4, 9 and 10 (Fig. 2). Eight months after presentation patient 2 had recurrent optic neuritis in the same eye. He was scanned again and found to have sphenoid sinus opacity. Post-treatment MRI scans were normal.

Patient 5 suffered intermittent attacks of visual loss that corresponded to episodes of sphenoid sinusitis over a period of 3 years. These attacks were initially treated medically. After surgical drainage she continued to 

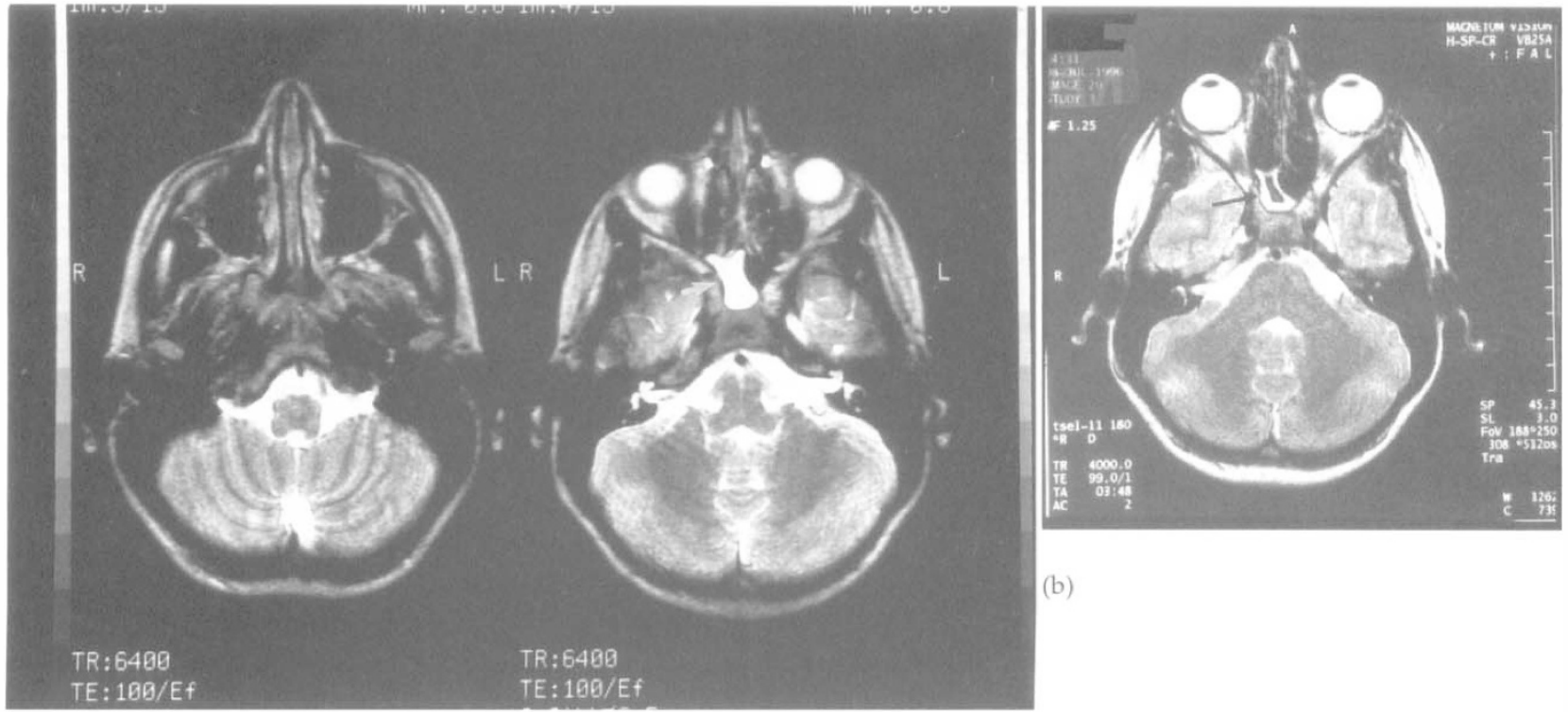

(a)

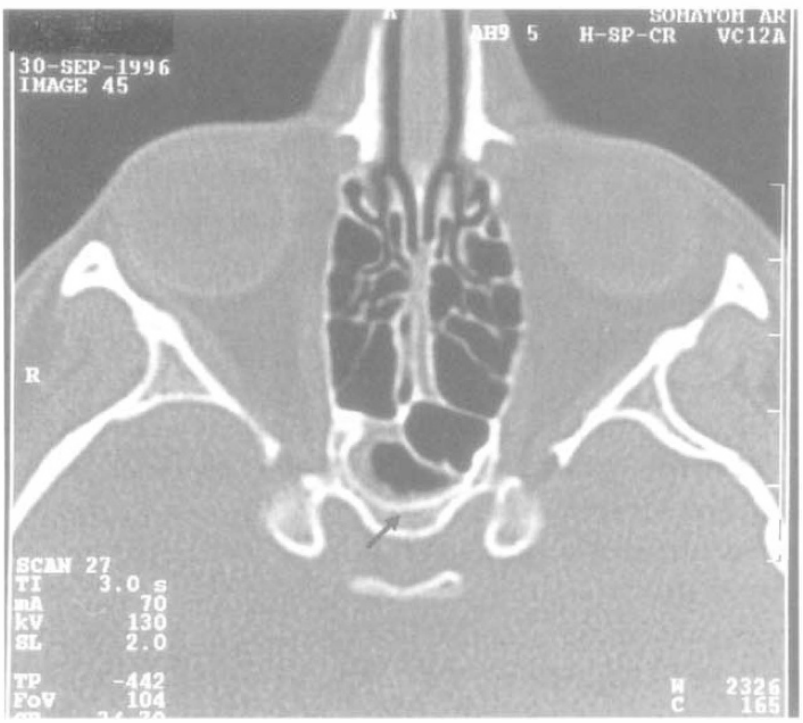

(c)

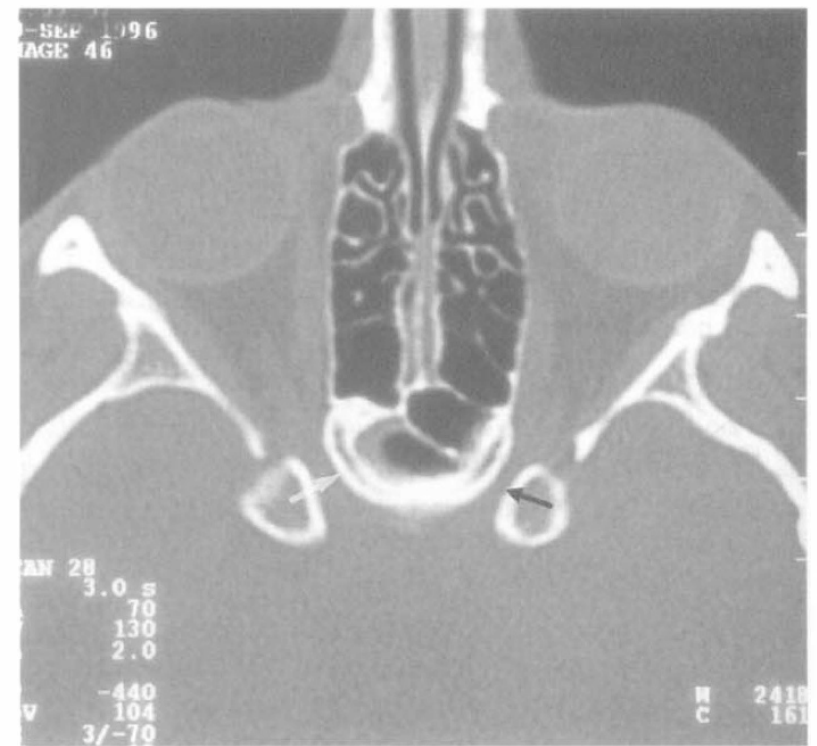

$(4)$

Fig. 1 (a) MRI scan of patient 1 at presentation in 1994. Note the sphenoid sinus opacity extending across the midline (arrow). (b) Repeat MRI scan in 1996, by which time both optic nerves were involved. The sphenoid sinus opacity has persisted (arrow). (c), (d) MRI scans showing extensive osteomyelitis of the wall of the sphenoid sinus (black arrow) causing narrowing of the optic canals, worse on the left (white arrow).

complain of occasional headache but her vision stabilised. Patient 6 had a prompt response to surgical drainage with resolution of the headaches and recovery of vision (Fig. 3). Patient 7 presented initially with isolated optic neuritis. Three weeks later he developed an ipsilateral partial third nerve palsy which, over the next few days, extended to include the fourth and sixth cranial nerves. An MRI scan showed a sphenoid sinus opacity that extended posteriorly to the cavernous sinus on the right side (Fig. $4 \mathrm{a}-\mathrm{c}$ ). Surgical drainage revealed widespread aspergillosis. The patient died of unrelated causes 3 months later.
Patient 8 also presented with an isolated optic neuropathy and atypical headache. Her sphenoid sinus opacity was found to be due to an invasive squamous cell carcinoma at surgery. She was referred for radiotherapy at her local hospital and lost to follow-up.

Patient 11, a 36-year-old man, recovered partially from his initial episode of optic neuritis and went on to develop optic neuritis in the fellow eye 1 year later. Three years after the initial episode he had reduced vision in both eyes and a repeat MRI scan was compatible with a diagnosis of multiple sclerosis. To date he is the only patient in the study known to have developed signs of generalised demyelinating disease (Fig. 5). 


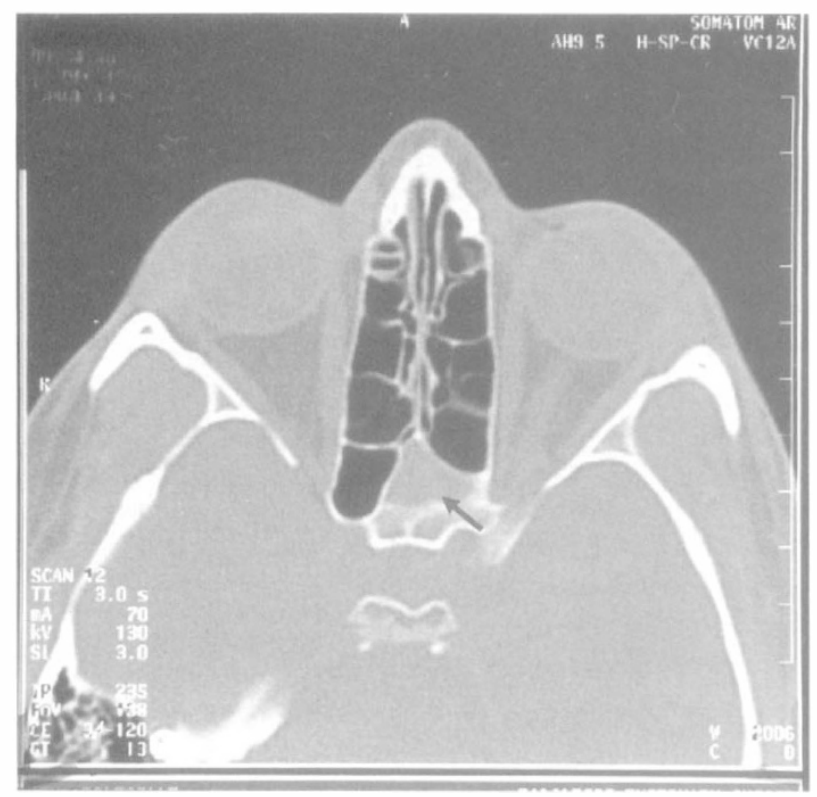

Fig. 2. Sphenoid sinus opacity (arrow) in a patient with decreased vision in the left eye and headache.

Our patients were followed-up for an average of 3 years (range 1-7 years).

\section{Discussion}

The mean age of patients recruited to the Optic Neuritis Treatment Trial is 31.8 years (range $18-50$ years). ${ }^{1}$ Eight of our patients were outside these limits ( 3 younger and 5 older), which suggested atypical features at presentation and was in part why they were investigated further. Although optic neuritis in the elderly has been found to behave similarly to the disorder in younger age groups, it

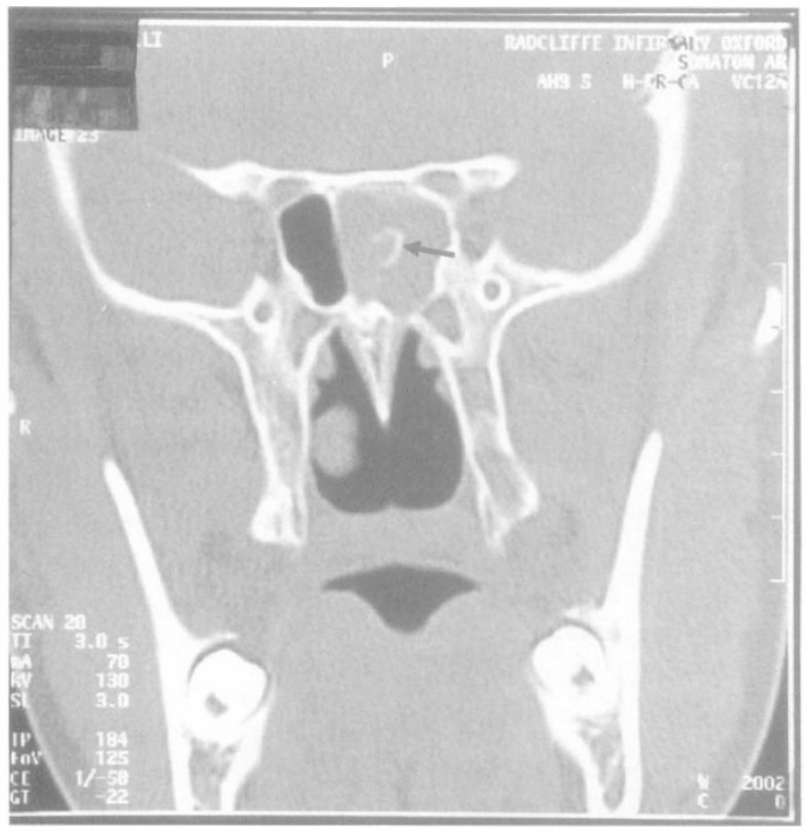

Fig. 3. MRI scan of a patient with aspergillosis involving the left sphenoid sinus. Note the calcification centrally, typical of fungal infection (arrow). is important to recognise specific aetiologies such as compression, ischaemia, infectious and infiltrative disorders. ${ }^{9}$ Of the 5 patients over the age of 50 years in our study, 2 had aspergillosis of the sphenoid sinus (extending to the cavernous sinus in 1 patient), 1 had a sinus tumour and 1 patient had polyps. The numbers in our study are small but it is possible to speculate that a sphenoid sinus opacity is even more significant in patients with optic neuritis outside the normal age range for the condition.

The Optic Neuritis Study Group has suggested that the 5 year cumulative probability of developing clinically definite multiple sclerosis (MS) in patients with optic neuritis is $30 \% .{ }^{10}$ Follow-up in our study was between 1 and 7 years, with the majority of patients being followed for at least 2.5 years and 4 patients for 4 or more years. We accept that this may not be long enough to exclude the development of MS as a final diagnosis in some patients. However, none of the 7 patients who had MRI scans were noted to have brain lesions at presentation and the Optic Neuritis Study Group also found that only a small minority (16\%) of such patients go on to develop MS in 5 years. ${ }^{10}$ Four patients had CT scan at presentation; ideally all patients should have had an MRI scan, but as this was a retrospective study and the cases were collected over several years, this was not possible. None of these patients has required further imaging.

Four patients had definite disc swelling at presentation; the appearance of the disc in patient 7 was thought to be equivocal. Patient 10 had proven sinus pathology at surgery (extensive polypoid tissue and pus). His MRI scan prior to surgery showed enhancement of the left optic nerve and anterior placement of the sphenoid sinus air cells surrounding the optic canal. This anatomical variant may have rendered the optic nerve more susceptible to involvement in the infectious process. Of the remainder, patient 3 was found to have pus in the sphenoid sinus at surgery and patients 2 and 9 were treated medically. It would seem possible that focal, chronic pathology in an anatomically vulnerable patient can cause swelling along the entire length of the optic nerve secondary to compression or inflammation. Alternatively it may be the case that the optic nerve head alone is affected due to arrested axoplasmic flow secondary to compression or inflammation of the optic nerve in the optic canal.

A causal relationship between paranasal sinus disease and acute optic neuritis was accepted in the historical literature. In 1909, Loeb presented an extensive literature review dating back to the early 1800 s and, in the absence of imaging, attributed many cases of visual loss to disease of the spheno-ethmoid sinuses; more recently there have been several case reports of optic neuritis associated with sphenoid sinusitis. ${ }^{11}$ With prompt antibiotic treatment and surgical drainage the condition was reversible, but delay in diagnosis and inappropriate treatment with steroids led to permanent visual loss. Acute isolated sphenoiditis accounts for less than $3 \%$ of all sinus infections. ${ }^{12}$. The most common symptom is headache, which can be retrobulbar, parietal or frontal; 


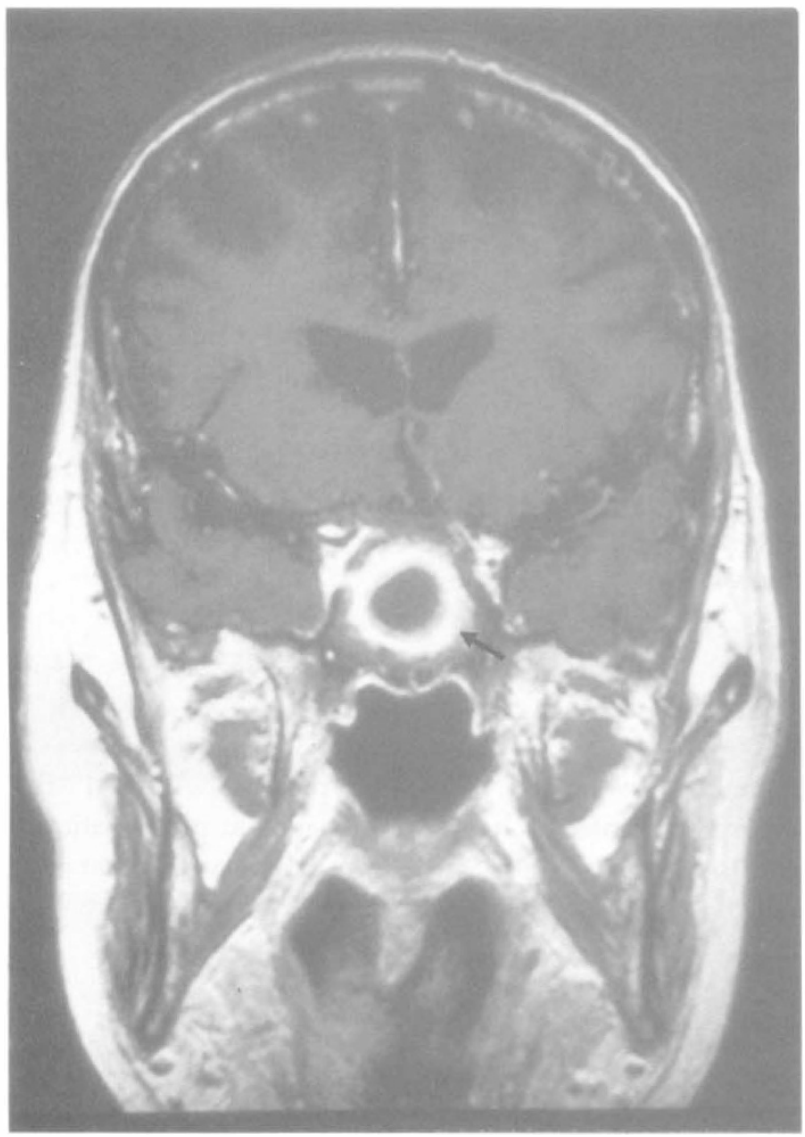

(a)

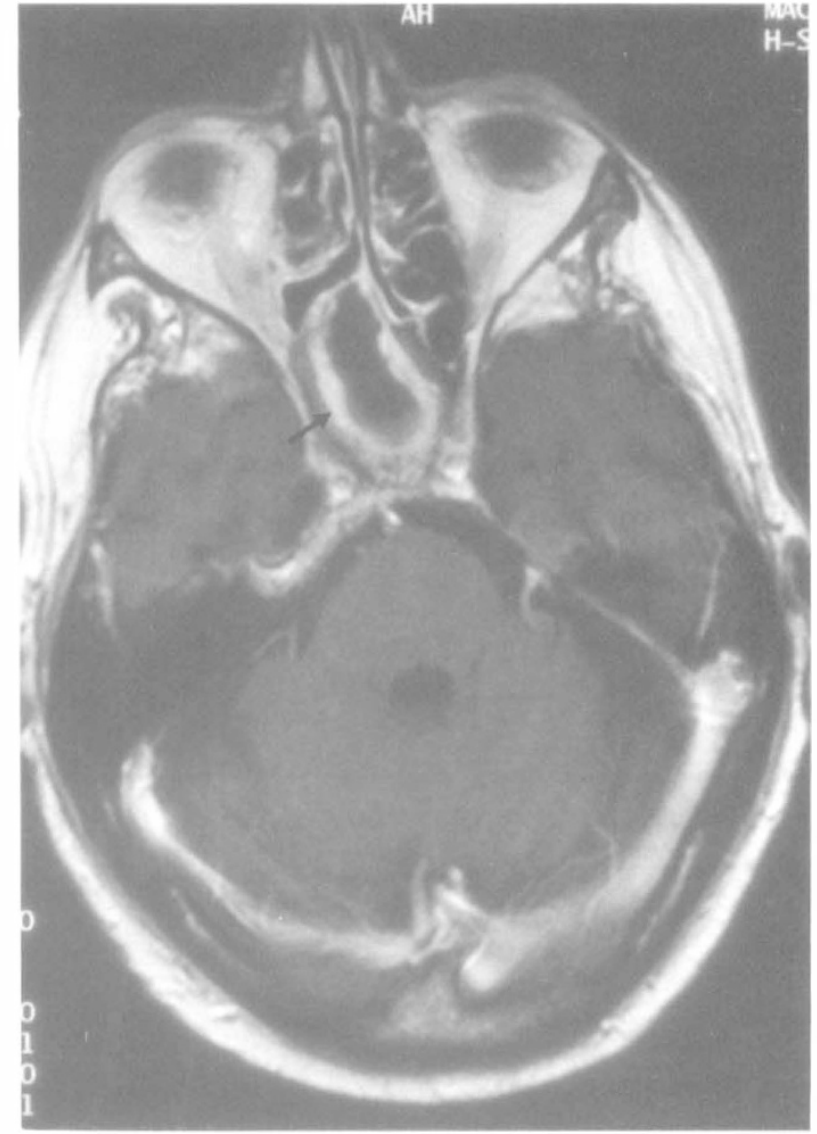

(b)

Fig. 4. (a) Gadolinium-enhanced T1-weighted MRI scan shows a thick rind of enhancement and central opacity in the sphenoid sinus extending up to the cavernous sinus on the right. (b) Axial view shows extensive changes in the right sphenoid sinus (arrow).

the pathological diagnosis is usually bacterial infection. ${ }^{13}$ Incidentally noted sphenoid sinus disease is uncommon. Digre et al. ${ }^{14}$ examined 300 consecutive, routine, neuroradiological scans and found sphenoid sinus opacity in only 21 (7\%): cases; in comparison the

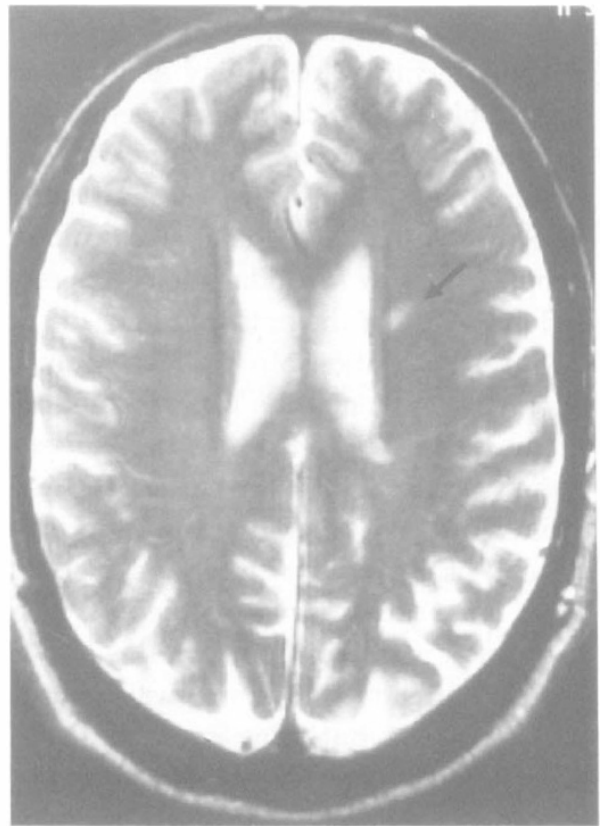

Fig. 5. Axial MRI scan of patient 11 showing multiple high-signal foci within the periventricular white matter (arrows). Appearances are consistent with multiple sclerosis. 
recovered fully after endoscopic sphenoidotomy. When present, therefore, opacity of the sphenoid sinus is likely to be significant.

All our patients were scanned because of one or more atypical features, i.e. age at presentation, systemic features, involvement of the fellow eye and/or the presence of headache as distinct from retrobulbar discomfort which is an accepted feature of optic neuritis. Suggested mechanisms of unilateral optic nerve involvement in the context of sphenoid sinus disease include direct spread of infection and inflammation, which are made possible by the anatomical features of the sphenoid sinus. Dissection of cadaveric heads has revealed an extremely variable relationship between the posterior spheno-ethmoid air cells and the optic nerve. Although the sphenoid sinuses are midline structures present in the body of the sphenoid bone, they can extend much further into the pterygoid processes, greater wing of the sphenoid or even encroach on the basilar part of the occipital bone. They are usually separated by a vertical median septum but the two sinuses are not symmetrical and one can lie in front of or behind the other. The bony wall covering the optic nerve is anatomically dehiscent in $6 \%$ of cases and, if present, may measure as little $0.10 \mathrm{~mm}$. In some cases the nerve can actually traverse the lumen of the sphenoid sinus. ${ }^{15}$ Osteomyelitis of the sinus walls secondary to chronic infection (as seen in patient 1) would then be in direct contact with optic nerve dural sheath. Subsequent thickening of the sinus wall would narrow the optic canal and lead to compressive optic neuropathy. Polyps involving the mucosa of the sphenoid sinus also cause compression of the nerve within the sinus.

Another possible mechanism of optic nerve involvement is invasion of the nerve by local bacteria in those cases where the nerve traverses the sphenoid sinus. Perhaps more likely is the possibility of secondary inflammatory occlusive vasculitis causing optic neuritis, which would explain the rapid resolution following surgical drainage and/or antibiotic therapy. A purely theoretical but intriguing possibility is the hypothesis that infection in the sphenoid sinus may stimulate the immune system indirectly causing demyelination; this could possibly explain the events in patient 11 .

A randomised controlled trial would be necessary to clarify the effects of medical or surgical intervention in these patients; optic neuritis presenting with sphenoid sinus disease is uncommon and in the absence of such data we feel our study, although not conclusive, highlights a number of important practical points:

1. Although data from the Optic Neuritis Treatment Trial conclude that MRI is not necessary in patients with typical features of optic neuritis, ${ }^{16}$ investigation is indicated in patients outside the normal age range, those with headache or systemic features, or a history of previous sinus disease, to exclude pathology involving the optic canal and posterior spheno-ethmoid sinuses. Interestingly we also reviewed the MRI scans of all the other patients with sphenoid sinus opacity and found no difference between patients with asymptomatic sphenoid sinus opacity found routinely and symptomatic sphenoid sinusitis with or without optic neuritis. However, the anatomical variations described would not be distinguishable on MRI scan and perhaps explain this finding.

2. In the context of visual loss an isolated opacity of the sphenoid sinus should not be ignored, as prompt treatment can restore vision in the affected eye. Complication rates of intranasal endoscopic sinus surgery range from $2 \%$ to $17 \%$, depending on the study reviewed. In experienced hands the overall complication rate is around $9 \%$, with the most common complications being synechia formation, orbital haematoma and antrostomy closure. More serious complications such as cerebrospinal fluid leak with meningitis, double vision and blindness are very rare; overall, endoscopic sphenoethmoidectomy is a safe procedure in experienced hands. ${ }^{17,18}$

3. Patient 1 developed intermittent optic neuritis in her fellow eye over a period of 16 months before undergoing surgical drainage of the sphenoid sinus. She was treated with oral steroids on a number of occasions with improvement in vision. We would suggest an MRI scan prior to commencing systemic steroids in patients with optic neuritis: otherwise underlying pathology such as infection and tumours can be masked and vision initially stabilise or even improve.

We conclude that the presence of sphenoid sinus opacity in patients with optic neuritis may well be significant and should not be dismissed as an identical finding.

\section{References}

1. Optic Neuritis Study Group. The clinical profile of optic neuritis: experience of the optic neuritis treatment trial. Arch Ophthalmol 1991;109:1673-8.

2. Ormerod IEC, et al. Disseminated lesions at presentation in patients with optic neuritis. J Neurol Neurosurg Psychiatry 1986;49:124-7.

3. Rothstein J, Maisel R, Berlinger N, Wirtschafter J. Relationship of optic neuritis to disease of the paranasal sinuses. Laryngoscope 1984;94:1501-8.

4. Sebst RG, Selhorst JB, Harrison JW, Myer EC. Parainfectious optic neuritis. Arch Neurol 1983;40:347-50.

5. Bobhen D, Sebag M, Michaud J. Paraneoplastic optic neuritis and encephalomyelitis. Arch Neurol 1988;45:353-6.

6. Naeser P. Optic nerve involvement in a case of methanol poisoning. Br J Ophthalmol 1988;72:778-81.

7. Loeb H. A study of the anatomic relations of the optic nerve to the accessory cavities of the nose. Ann Otol Rhinol Laryngol 1909;18:243.

8. Onodi N. The optic nerve and the accessory cavities of the nose. Ann Otol Rhinol Laryngol 1908;7:1.

9. Jacobson DM, Thompson HS, Corbett JJ. Optic neuritis in the elderly: prognosis for visual recovery and long-term followup. Neurology 1988;38:1834-7.

10. Optic Neuritis Study Group. The five-year risk of MS after optic neuritis. Neurology 1997;49:1404-13.

11. Lew D, Southwick FS, Montgomery WW, et al. Sphenoid sinusitis: a review of 30 cases. $N$ Engl J Med 1983;309:1149-54.

12. Deans JAJ, Welch AR. Acute isolated sphenoid sinusitis: a disease with complications. J Laryngol Otol 1991;105:1072-4. 
13. Hadar T, Yaniv E, Shvero J. Isolated sphenoid sinus changes: history, CT and endoscopic finding. J Laryngol Otol 1996;110:850-3.

14. Digre K, Maxner C, Crawford S, Yuh W. Significance of CT and MRI findings in sphenoid sinus disease. AJNR 1989;10:603-6.

15. Yeoh $\mathrm{K}$, Tan $\mathrm{K}$. The optic nerve in the posterior ethmoid in Asians. Acta Otolaryngol 1994;114:329-36.
16. Beck R, Arrington J, Murtagh R, Cleary P, Kaufman D. Optic neuritis study group. Brain magnetic resonance imaging in acute optic neuritis. Arch Neurol 1993;50:841-6.

17. Stankiewicz JA. Complications in endoscopic intranasal ethmoidectomy: an update. Laryngoscope 1989;99:686-90.

18. Stankiewicz JA. Complications of endoscopic sinus surgery. Otolaryngol Clin North Am 1989;22:749-58. 